\title{
Oxygen Saturation Trend in Normal Healthy Term Newborn
}

\author{
Dr. Sunil Gavhane ${ }^{1 *}$, Dr. Jagruti Mahale ${ }^{2}$, Dr. Sachin Dhule ${ }^{3}$, Dr. Madhuri Engade ${ }^{4}$, Dr. Harshal Tukaram Pandve ${ }^{5}$
}

\author{
${ }^{1}$ Associate Professor, Dept. of Pediatrics, MGM Medical College, Aurangabad, Maharashtra, India \\ ${ }^{2}$ Senior Resident, Dept. of Pediatrics, MGM Medical College, Aurangabad, Maharashtra, India \\ ${ }^{3}$ Assistant Professor, Dept. of Pediatrics, MGM Medical College, Aurangabad, Maharashtra, India \\ ${ }^{4}$ Associate Professor, Dept. of Pediatrics, MGM Medical College, Aurangabad, Maharashtra, India \\ ${ }^{5}$ Professor and Head, Dept. of Community, Medicine, ESIC Medical College, Sanathnagar, Hyderabad, Telangana, India
}

DOI: $10.36347 / \mathrm{sajb} .2020 . \mathrm{v} 08 \mathrm{i} 07.001$

| Received: 26.06.2020 | Accepted: 03.07.2020 | Published: 08.07.2020

*Corresponding author: Dr. Sunil Gavhane

\section{Abstract}

Original Research Article

Background: An infant after birth goes transition from foetal circulation to neonatal circulation. Changes in ventilation and circulation occur during this time indicated by the changes in heart rate, respiratory rate and APGAR score. Aim: To form a normative data of pulse oxymetry readings in newborns at $1 \mathrm{st}$, 5th, and 10th minute of life, in healthy term newborn. Material Methods: The study was conducted in tertiary care hospital in India during the period of 2 years. All the full term healthy newborns and no resuscitation required at birth were included in the study after taking voluntary informed consent of their parents. Predesigned semi-structured questionnaire was prepared based on the review of literature on pulse oximetry in healthy newborns Results: Total 2873 babies out of 4326 born were included in the study. Mean heart rate was 139 per minute at 1 minute which gradually reduced to 122 per minute at the end of 10 minutes. Mean respiratory rate was 45 per minute at 1 minute which gradually reduces to 40 per minute at the end of 10 minutes. Mean APGAR score was 9 at 1 minute which increased to 10 at the end of 10 minutes. Mean SpO2 was $67 \%$ at 1 minute which increased to $83 \%$ at the end of 5 minutes and $91 \%$ at the end of 10 minutes of life. Conclusion: the study concluded saturation trends didn't vary according to the gender, birth weight in term infants.

Keywords: Oxygen saturation, Term neonate, Pulse oxymetry.

Copyright @ 2020: This is an open-access article distributed under the terms of the Creative Commons Attribution license which permits unrestricted use, distribution, and reproduction in any medium for non-commercial use (NonCommercial, or CC-BY-NC) provided the original author and source are credited

\section{INTRODUCTION}

Initial few minutes are the most important and vulnerable period in a newborn's life as there is transformation of fetal circulation into the newborn circulation [1]. Along with all this the oxygen saturation of the newborn improves from an intrauterine Spo2 of $40 \%$ to $90 \%$ [4]. In this study normative ranges of Spo2 at $1^{\text {st }}, 5^{\text {th }}$, and $10^{\text {th }}$ minute of life in the healthy newborns born at our centre have been described. Pulse oxymetry is one of the noninvasive, reliable and cost effective tools for assessing the perfusion and the oxygen saturation [2].

\section{MATERIALS AND METHODS}

A cross sectional study was conducted in tertiary care centre in India during the period of 2 years. All the full term healthy newborns (gestational age more than or equal to 37 weeks) and no resuscitation required at birth were included in the study after taking voluntary informed consent of their parents. Infants with hydrops fetalis, meconium stained amniotic fluid, respiratory distress or any gross congenital malformations were excluded. Predesigned semi-structured questionnaire was prepared based on the review of literature on pulse oximetry in healthy newborns which included gender of baby, gestational age and age of mother, gravid status, type of delivery, birth weight, clinical symptoms, blood pressure measurement, APGAR score at 1 and $5 \mathrm{~min}$, congenital anomaly, antenatal illness, intrapartum risk factors and pulse oximetry readings at $1^{\text {st }}, 5^{\text {th }}$ and $10^{\text {th }}$ minutes.

A Nelcor technology based with silicone probe pulse oxymeter was used for the study. Probe was attached to the right wrist of the newborn immediately after receiving the newborn in the warmer and drying. Readings were taken at $1^{\text {st }}, 5^{\text {th }}, 10^{\text {th }}$ minute of life once plethysmograph is stable and uniform.

\section{Statistical Analysis}

Data management and analysis was done using Microsoft excel and Epi-info software. The frequency distribution and graph were prepared for the variables. The categorical variables were assessed using Pearson chi-square test. 


\section{Ethical Considerations}

The study protocol was approved by the Scientific and Ethical Committee of the Institution. All the participants' parent was informed about the study procedure and the information required from them for the study. A voluntary informed written consent was taken from the participant parent those who consented were included in the study. A strict confidentiality was maintained about the personal details of the participants and information related to the study.

\section{RESULTS}

Among the total 4326 live birth occurred in the institute of which 2873 newborns were included in the study fulfilling the inclusion and exclusion criteria.

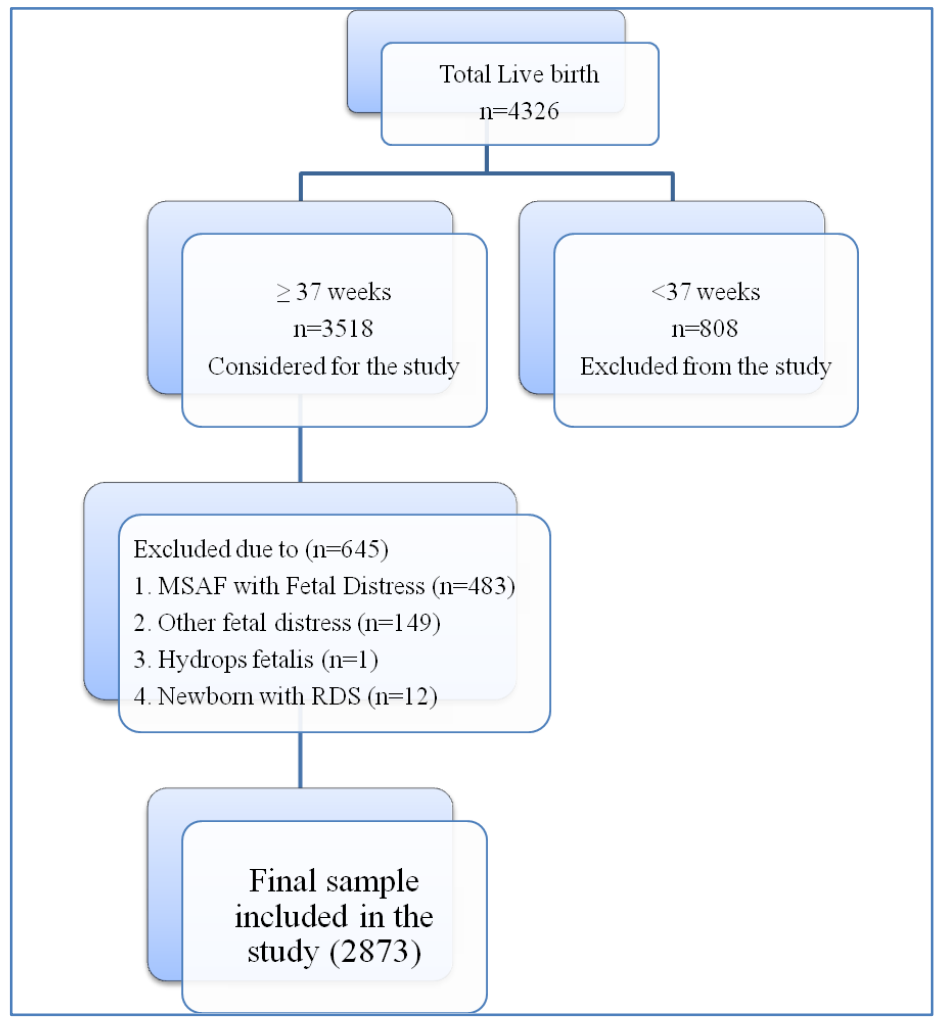

Fig-1: Sample inclusion of study

The observations showed the significant difference ( $\mathrm{p}$ value $<0.01$ ) in the mean $\mathrm{SpO} 2$ value at first min between the vaginally born and newborns born by LSCS which decreased till tenth minute of life (fig no. 1) The mean saturation of newborns born vaginally at 1 min was $70+\_7.3 \%$ and $63+\_7.5 \%$ born by cesarean section. No significant difference between the SpO2 readings between the newborns born between 37 to 39 weeks of gestation and the newborns with gestational age more than 39 weeks between the two was observed ( $\mathrm{p}>0.05$ ) ( fig no.2).

The mean $\mathrm{SpO} 2$ was $67 \%$ at 1 minute which increased to $91 \%$ at the end of 10 minutes. The rate of change in $\mathrm{SpO} 2$ was statistically significant $(\mathrm{p}<0.01)$.
SPO2 values according to birth weight indicate that the SPO2 level was not significantly lower in low birth weight babies compared to normal weight babies ( $p$ $>0.05$ ) as shown in table no. 1 .

The mean heart rate was 139 per minute at 1 minute which reduced to 122 per minute at the end of 10 minutes; mean respiratory rate was 45 per minute at 1 minute which reduced to 40 per minute at the end of 10 minutes as shown in fig no. 3 .

$1^{\text {st }}$ minute saturation values according to gender indicate that the saturation level was non-significantly lower in females compared to male babies (as p >0.05) as shown in fig no.3.

Table-1: Saturation trends in low birth weight newborns and newborns above or equal to 2500gm

\begin{tabular}{|l|l|l|l|l|l|}
\hline \multirow{2}{*}{ Birth weight $($ gms) } & \multirow{2}{*}{ No. of Newborn } & \multirow{2}{*}{ Percentage } & \multicolumn{3}{|l|}{ Mean saturation at } \\
\cline { 3 - 6 } & & & $\mathbf{1}^{\text {st }}$ min & $\mathbf{5}^{\text {th }} \mathbf{m i n}$ & $\mathbf{1 0}^{\text {th }}$ min \\
\hline$<2500$ & 713 & $24.82 \%$ & $64 \pm 7.7$ & $83 \pm 7.2$ & $90 \pm 6.8$ \\
\hline$\geq 2500$ & 2160 & $75.18 \%$ & $69 \pm 7.4$ & $86 \pm 7.3$ & $91 \pm 6.8$ \\
\hline Total & 2873 & $100.00 \%$ & 67 & 85 & 91 \\
\hline
\end{tabular}




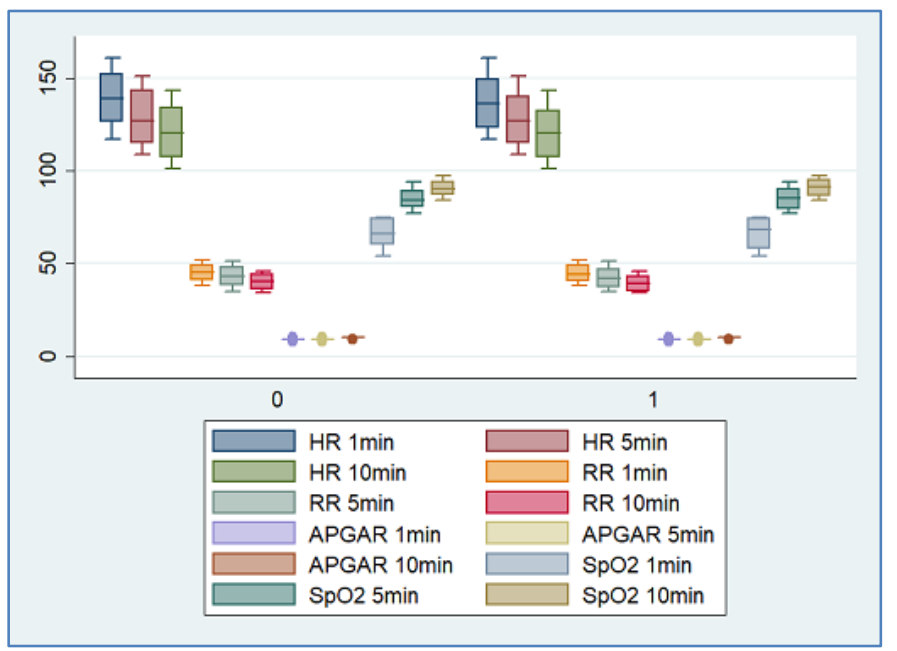

Fig-2: Box whisker plot showing variables against mode of delivery, LSCS=0, NVD=1

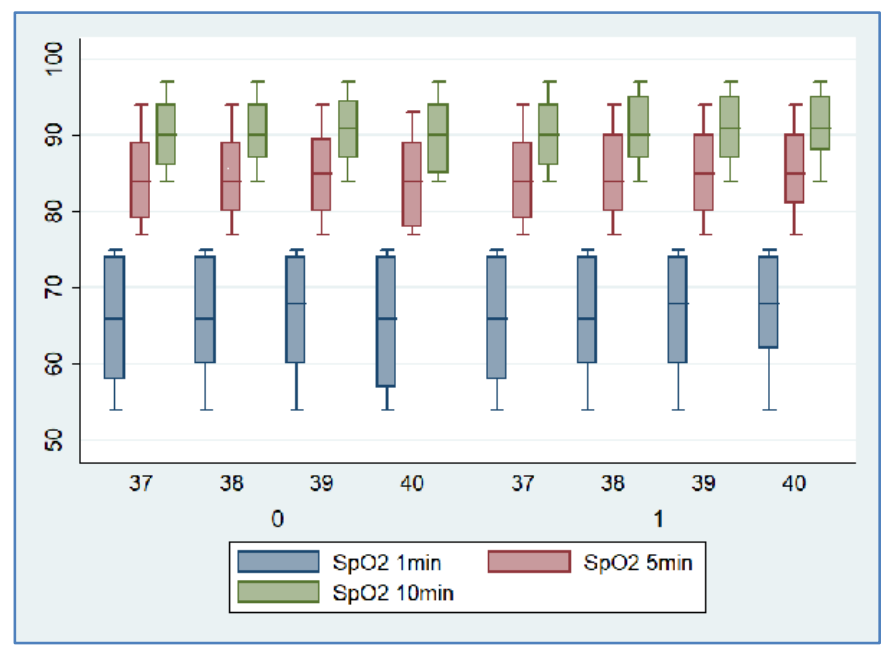

Fig-3: Box whisker diagram showing Spo2 (y axis) against period of gestation POG on $x$ - axis

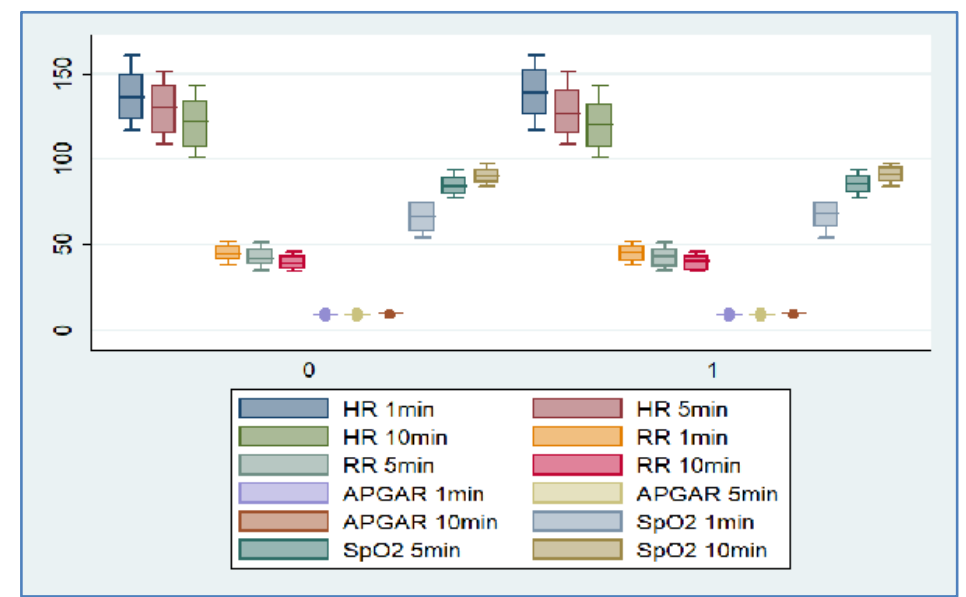

Fig-4: Box whisker showing variable against gender, male-1, female-2

\section{DISCUSSION}

We have used Nelcor pulse oximeter with a neonatal silicon probe which is easily available in rural and remote areas wherease latest oxygen saturation measuring technology including signal extraction technology, masimo are costly and not easily available. Readings to be taken at $1^{\text {st }}, 5^{\text {th }}, 10^{\text {th }}$ minute of life. Present study was aimed towards obtaining normative data for the term newborns. We studied the trends of rise in saturation in newborns with gestational age 37-39 weeks and >39 weeks separately which did not show any significant difference in mean saturations at $1^{\text {st }}, 5^{\text {th }}$ and $10 \mathrm{~min}$ (as p-value $>0.05$ ). 
The European Resuscitation Council guidelines advise to start resuscitation in term infants with air rather than $100 \%$ oxygen and to follow oxygen saturation (SpO2) targets for the first $10 \mathrm{~min}$ after birth. These targets are based on observational studies by Dawson et al. [2]. They measured $\mathrm{SpO} 2$ in the first $10 \mathrm{~min}$ after birth in 468 infants who received no medical intervention in the delivery room. These data were used to develop reference ranges for $\mathrm{SpO} 2$ in the first $10 \mathrm{~min}$ after birth for preterm and term infants i.e. median oxygen saturations rise steadily from around $60 \%$ at 1 min of age to above $90 \%$ by $10 \mathrm{~min}$ [2]. From this observational study we got mean Spo2 levels of the healthy newborns born which will be helpful during resuscitation.

In the study conducted by Donia et al. [3] the postductal mean $\mathrm{SpO} 2$ value was $92 \pm 1 \%$ (range: $88-$ 94\%) and $93 \pm 1 \%$ (range: $91-94 \%$ ) at the initial and repeat tests respectively. S Richmond et al. [4] made use of pulse oxymetry as a screening test for critical congenital heart diseases along with ANC scans and clinical assessment at birth.

Harris et al. [5] found the mean $\mathrm{SpO} 2$ at $1 \mathrm{~min}$ was $46 \%(3 \%)$ in the caesarean group and $61 \%(5 \%)$ in the vaginal delivery group $(\mathrm{p}, 0.05)$, but by $5 \mathrm{~min}$ there was no significant difference. This difference in saturation decreased at $5 \mathrm{~min}$ and was insignificant at 10 min (as p>0.05), same finding was observed in present study in relation to heart rate, respiratory rate. Kamlin et al. [6] found that term infants born by elective caesarean section took on average 2 min longer to reach an $\mathrm{SpO} 2$ of $90 \%$ than infants born by spontaneous vaginal delivery.

In the study by Kamlin et al. [6] on infants not receiving resuscitation, the time to reach an $\mathrm{SpO} 290 \%$ was significantly longer in 54 preterm infants at 6.5 (4.9-9.8) min (median (IQR)) than in 121 term infants at 4.7 (3.3-6.4) min (median (IQR)) (p-0.001).

Deckardt et al. [7] observed the effect of oximetry during resuscitation in 15 infants of $<30$ weeks' gestation. CPAP was used only if the $\mathrm{SpO} 2$ was $<80 \%$ at $5 \mathrm{~min}$ and stopped once the $\mathrm{SpO} 2$ reached $90 \%$. (FiO2) was reduced from 1.0 to average 0.40 with help of oximetry [7].
In the study conducted by Habib et al. [8] the rate of change in APGAR Score was statistically significant $(\mathrm{p}<0.01)$ which indicate that in newborn APGAR score significantly improves within first 10 minutes of life which is consistent with our finding.

\section{CONCLUSION}

From the study we concluded that the mean spo 2 of a newborn at $1^{\text {st }}, 5^{\text {th }}$, and $10^{\text {th }}$ minute was $67 \%$, $83 \%$, and $91 \%$ respectively. From the study we also found out that there was a difference in mean saturation levels at $1^{\text {st }}, 5^{\text {th }}$, and $10^{\text {th }}$ minute between vaginally born and those born by ceserean section. Saturation trends didn't vary according to the gender, birth weight in term infants.

\section{REFERENCES}

1. Dawson JA, Kamlin CO, Vento M, Wong C, Cole TJ, Donath SM, Davis PG, Morley CJ. Defining the reference range for oxygen saturation for infants after birth. Pediatrics. 2010 Jun 1;125(6):e1340-7.

2. Ewer AK, Middleton LJ, Furmston AT, Bhoyar A, Daniels JP, Thangaratinam S, Deeks JJ, Khan KS, PulseOx Study Group. Pulse oximetry screening for congenital heart defects in newborn infants (PulseOx): a test accuracy study. The Lancet. 2011 Aug 27;378(9793):785-94.

3. Donia A, Tolba O. Use of early pulse oximetry in the detection of cardiac lesions among asymptomatic term newborns. Egyptian Pediatric Association Gazette. 2016: 64:1-5.

4. Richmond S, Reay G, Harb M. Routine pulse oximetry in the asymptomatic newborn. Arch Dis Child Fetal Neonatal Ed 2002;87:F83-F88

5. Harris P, Sendak J, Donham T. Changes in arterial oxygen saturation immediately after birth in the human neonate. J Pediatr. 1986;109:117-19.

6. Kamlin O, Donnell F, Davis G. Oxygen saturation in healthy infants immediately after birth. J Pediatr. 2006;148:585.

7. Donnell F, Kamlin O, Davis G. Endotracheal intubation attempts during neonatal resuscitation: success rates, duration, and adverse effects. Pediatrics. 2006;117:e16-21.

8. Habib S. Oxygen saturation trends in the first hour of life in healthy full-term neonates born at moderate altitude. Pak J Med Sci. 2013;29(4):903-906. 